\title{
Roland Barthes im Gespräch mit Georges Charbonnier: Über eine mögliche Theorie der Lektüre (1967)
}

\author{
Prof. Dr. Sandro Zanetti \\ Universität Zürich, Romanisches Seminar/AVL, Plattenstrasse 43, \\ $\mathrm{CH}-803_{2}$ Zürich \\ sandro.zanetti@uzh.ch \\ Dr. Agathe Mareuge \\ Universität Zürich, Romanisches Seminar/AVL, Plattenstrasse 43, \\ $\mathrm{CH}-8032$ Zürich \\ agathe.mareuge@uzh.ch
}

\section{Vorbemerkung}

Im Oktober 1967 führte der Literaturkritiker Georges Charbonnier mit Roland Barthes eine Reihe von Gesprächen, die noch im selben Monat in Form von sechs jeweils rund zwanzigminütigen Rundfunksendungen auf France Culture ausgestrahlt wurden. Die hier wiedergegebene fünfte Sendung vom 24 . Oktober 1967 trug den Titel „Énonciation, intersubjectivité, intertextualité et analyse psychanalytique et linguistique de la lecture". Transkription und Übersetzung folgen der Originalaufnahme von 1967 , wobei die letzten rund fünfeinhalb Minuten der Sendung, die vom Thema der Lektüre wegführen, ausgelassen wurden. 1988 wurde in der Reihe $\grave{A}$ voix nue das gesamte Tonmaterial noch einmal neu zusammengestellt und in Form von fünf Sendungen ausgestrahlt. Die Originalaufnahmen von 1967 sowie vier der fünf erweiterten und bearbeiteten Sendungen von 1988 sind seit Anfang 2016 auf der Website von France Culture frei zugänglich. ${ }^{1}$

Die von Roland Barthes gesprächsweise formulierten Überlegungen zu einer Theorie der Lektüre fallen in die Zeit des wirkungsmächtigen Aufsatzes „La mort de l'auteur“ („Der Tod des Autors“). Der Aufsatz erschien ebenfalls

$1 \mathrm{http}: / / \mathrm{www}$.franceculture.fr/2016-o1-19-a-voix-nue-roland-barthes-entretiens-originaux -de-roland-barthes-avec-georges-charbonnier\# bzw. https://www.franceculture.fr/dossiers/ roland-barthes-l-integrale-en-cinq-entretiens-1967 [letzter Zugriff 05.02.2018]. 
1967 - zuerst im Dezember in einer englischen Übersetzung, im Jahr darauf dann auf Französisch. Der Aufsatz endet mit dem berühmt gewordenen polemischen Satz: „La naissance du lecteur doit se payer de la mort de l'Auteur.“ („Die Geburt des Lesers ist zu bezahlen mit dem Tod des Autors.“) Der Wechsel in der Aufmerksamkeit von den Aktivitäten des Autors zu jenen des Lesers - einschließlich jenen des Autors als Leser - ist auch Thema des hier wiedergegebenen Gesprächs mit Georges Charbonnier. Deutlich zu spüren ist die Geste der Abgrenzung, mit der Barthes auch und gerade in seinen ersten, noch tastenden Überlegungen zu einer Theorie der Lektüre von der Fixierung auf die Figur und die Intentionen des Autors loskommen möchte. Dabei weist er die Instanz des Autors selbstbewusst bereits einer vergangenen Epoche zu. Gleichzeitig lenkt er die Aufmerksamkeit weg vom empirischen Leser hin zum Akt der Lektüre sowie, noch wichtiger, zur Frage, wie Texte und ,Lektüresubjekte sich zueinander strukturell verhalten.

Vorbereitet wurden die im Gespräch mit Georges Charbonnier formulierten Überlegungen bereits in Barthes' literaturkritischen Schriften der Fünfziger- sowie der frühen und mittleren Sechzigerjahre, insbesondere in der Streitschrift Critique et vérité von 1966. Kurz nach den Rundfunkgesprächen beginnt Barthes außerdem sein mehrsemestriges Seminar zu Balzacs Erzählung Sarrasine an der École pratique des hautes études. Das Seminar ist die direkte Vorarbeit und Vorbereitung des Buches $S / Z$, das 1970 erscheint und selbst Beispiel einer neuen, strukturalistischen Art der Lektüre ist. Die „Geburt des Lesers" trägt nun auch methodologisch Früchte, indem Barthes konsequent nicht den Autor (Balzac) in den Vordergrund stellt, sondern den Text in seinen intertextuellen Verflechtungen sowie in seiner spezifischen Organisation und Vernetzung sprachlicher Codes. Den auch in den Gesprächen mit Charbonnier verwendeten Begriff der ,Intertextualität' übernimmt Barthes von Julia Kristeva, die ihn - ebenfalls 1967 - in ihrem Aufsatz „Bakhtine, le mot, le dialogue et le roman“ einführte. Ein Jahr zuvor, Ende 1966, stellte Kristeva ihre Überlegungen zur Intertextualität, die sie aus ihrer Auseinandersetzung mit den Schriften Michail Bachtins zur Dialogizität gewonnen hatte, im Seminar von Barthes vor. Mit dem Begriff der „écriture-lecture“ schlug sie zugleich eine Brücke zu den u.a. von Barthes vorangetriebenen Forschungen zur „écriture“ - zur Schrift und zum Schreiben, die beide nun verstärkt unter dem Gesichtspunkt der darin implizierten Lektüreaktivitäten konzeptualisiert wurden.

Für Barthes und seine Überlegungen zu einer Theorie der Lektüre ist der Begriff der ,Intertextualität' von entscheidender Bedeutung, macht er es doch möglich, den ,Text' selbst als ,Geflecht' produktiver Lektüren zu definieren. Mit anderen Worten: Der Textbegriff selbst impliziert in seiner Struktur eine jeweils spezifische Verflechtung mit Intertexten, die wiederum als Effekte produktiver Lektüren bestimmt werden können. Insbesondere in der deutschsprachigen 
Diskussion ist Barthes ebenso wie Kristeva des Öfteren der Vorwurf gemacht worden, mit einem unscharfen Begriff von Intertextualität zu operieren, der es kaum möglich mache, verschiedene Formen, historische Manifestationen und Explizitheitsgrade von Intertextualität zu unterscheiden. Man muss sich allerdings vergegenwärtigen, dass Barthes wissenschaftsgeschichtlich ganz am Anfang einer Diskussion zur Erforschung von Lektüreprozessen stand: Die maßgeblichen Schriften etwa der ,Konstanzer Schule‘ zur Rezeptionsästhetik erschienen erst später, die Arbeiten aus dem Umkreis des Reader-response criticism waren nur punktuell rezipiert, und auch Umberto Ecos Lector in fabula (1979) - obschon vorbereitet durch die Studie Opera aperta von 1962 - war noch nicht erschienen. Zugleich zeigte sich Barthes allerdings, wie auch in den folgenden Ausführungen, von Anfang an skeptisch gegenüber einer Theorie des Lesens oder des Lesers, die sich entweder - durch traditionelle Einflussforschung oder Literaturpsychologie - weiterhin auf die Instanz des Autors (was hat er gelesen und warum?) oder - durch empirische Literatursoziologie - auf die Literaturgeschichte im Sinne einer Rezeptionsgeschichte bzw. auf statistische Erhebungen realer Lesererfahrungen konzentriert.

Stattdessen rückt Barthes konsequent den Gedanken ins Zentrum, dass es der Text selbst ist, der als ein Geflecht produktiv gewordener und werdender Lektüren zu bestimmen und zu erörtern ist. Darin involviert ist das ,Lektüresubjekt', das von Barthes primär sprachlich gedacht wird. Das ,Schreibsubjekt ${ }^{\star}$ wiederum wird von ihm so bestimmt, dass es immer auch ein ,Lektüresubjekt ${ }^{\star}$ ist - während umgekehrt das ,Lektüresubjekt' schreibend' in dem Sinne ist, dass es den Text aktiv weiterspinnt. Um diese Relationen im Einzelnen beschreiben und systematisieren zu können, schlägt Barthes eine Kooperation zwischen Linguistik, Literaturwissenschaft und Psychologie bzw. Psychoanalyse vor. Vieles bleibt in diesen Überlegungen noch ungeklärt und offen. Aus heutiger Perspektive mag deren Reiz jedoch gerade darin bestehen, dass sie von Barthes im Status des Unfertigen, erst Angedachten und Improvisierten vorgetragen werden. Man hat es hier also mit einer Theorie im Entwurf zu tun: mit ersten Erwägungen, Sondierungen und Grenzziehungen, die von Barthes später teilweise wieder verworfen werden, in anderen Zusammenhängen aber auch produktive Fortsetzungen finden.

So weisen etwa die hier gegen Ende vorgetragenen Überlegungen zum hedonistischen, erotischen, lustvollen Lesen bereits vor auf Le Plaisir du texte von 1973. Der Wechsel vom ,strukturalistischen' zum ,poststrukturalistischen Roland Barthes ist gerade mit Blick auf die offenen Wege, die Barthes in den Gesprächen, aber auch in seinen Seminaren wählt, weniger eindeutig zu bestimmen, als die publizierten Schriften nahelegen könnten. Auch Barthes' Hinwendung zur Lust am Text hat seine Vorgeschichte, auf die im Folgenden sogar explizit hingewiesen wird: die Auseinandersetzung mit der antiken 
Rhetorik. Bereits 1964 stellt Barthes im Vorwort zu seinen Essais critiques bündig fest, dass die Rhetorik „die amouröse Dimension der Schrift“ sei („La rhétorique est la dimension amoureuse de l'écriture“). Später, um 1970, versammelt er seine Notizen zur antiken Rhetorik in der einschlägigen ,Gedächtnisstütze „L'ancienne rhétorique. Aide-mémoire“.

Das Thema des Lesens wiederum bleibt ein konstanter Bezugspunkt in Barthes' Arbeiten, die selbst stets praktische Verknüpfungen von Lese- und Schreibtätigkeiten sind. Diese Verknüpfungen reichen von der „sinnlichen Lektüre" in L'Empire des signes (1970) bis zur Erprobung und Reflexion neuer Lesepraktiken in S/Z (1970) und Le Plaisir du texte (1973), sie weisen aber auch zurück und nach vorn bis zu jenen Lesevorgängen, die sich auf das Feld der Literatur und der Schrift nicht beschränken lassen (Fotografie, Mode, kulturelle Objekte und Prozesse im weitesten Sinn).

Transkription und Übersetzung des Gesprächs mit Georges Charbonnier von 1967 folgen der auf der Website von France Culture bereitgestellten Tonaufnahme. Der mündliche Duktus wurde beibehalten, ohne indessen darauf zu verzichten, einen möglichst gut lesbaren Text darzubieten. ${ }^{2}$

\section{Transkription und Übersetzung}

GC

Roland Barthes, une théorie de l'écriture est en voie d'élaboration, elle verra le jour, mais il n'existe pas encore de théorie du lecteur. Peut-on imaginer une théorie du lecteur?

$\mathrm{RB}$

On peut l'entrevoir d'une façon qui restera encore très hermétique et très métaphorique. Si vous voulez, on peut la concevoir d'une façon d'abord un petit peu négative; je suis persuadé que la théorie de la lecture, la théorie du lecteur, si on peut l'édifier un jour, ne pourra pas être édifiée à partir de réflexions ou d'interrogations de type
GC

Roland Barthes, eine Theorie der Schrift und des Schreibens (écriture) wird zurzeit gerade ausgearbeitet, sie wird das Licht der Welt erblicken. Eine Theorie des Lesers (théorie du lecteur) hingegen gibt es noch nicht. Kann man sich eine Theorie des Lesers vorstellen?

RB

Man kann sie auf eine Weise erahnen, die aber noch sehr hermetisch und metaphorisch bleiben wird. Man kann sie zunächst, wenn Sie so wollen, auf eine etwas negative Weise erfassen. Ich bin überzeugt davon, dass die Theorie des Lesens, die Theorie des Lesers, wenn es sie eines Tages geben sollte, nicht von Überlegungen oder Befragungen

2 Der Abdruck erfolgt mit freundlicher Genehmigung von France Culture, Éric Marty und Jean Charbonnier. 
sociologique, c'est-à-dire [que] ça n'est pas en se demandant par différents moyens d'enquête par exemple 'qu'est-ce qui se passe dans la tête du lecteur ou de cent lecteurs ou de mille lecteurs quand ils lisent un texte', ce n'est pas en essayant de savoir cela...

\section{GC}

...or c'est ce genre d'enquêtes que l'on mène actuellement...

\section{$\mathrm{RB}$}

...bien sûr; ce n'est pas par une sociologie de la lecture qu'on arrivera à savoir ce que c'est que la lecture au niveau du texte. On y arrivera je crois par, si vous voulez, à la fois une linguistique et une psychologie renouvelées, peut-être même une psychanalyse dans la mesure où la psychanalyse est en train de bouger tout de même actuellement, [c'est par là] qu'on pourra édifier ou proposer une sorte de théorie générale de la lecture. Ce serait déjà un point énorme si on admettait et si on tirait toutes les conséquences de ce fait, à savoir que de même qu'il y a un sujet de l'écriture qu'on appelait jusqu'à présent l'auteur', n'est-ce pas, eh bien il y a aussi au fond un sujet au sens plein de la lecture. On peut imaginer ou postuler un certain nombre de caractères, de traits de ce sujet de la lecture - ce sujet de la lecture qui ne doit pas être pensé comme une personne finie, comme une identité finie, n'est-ce pas, douée par conséquent d'une épaisseur sociologique, classe d'âge, classe socioprofessionnelle etc., mais comme au fond ce que nous pensons maintenant du sujet, le sujet c'est est lui-même un langage, si vous voulez, et alors en ce sens on peut dire que le soziologischer Art ausgehen kann. Das heißt, es wird nicht darum gehen können, beispielsweise über verschiedene Umfragen oder Untersuchungen zu erfahren, was sich im Kopf des Lesers abspielt oder im Kopf von hundert oder tausend Lesern, wenn diese einen Text lesen. Es geht nicht darum, dies wissen zu wollen... GC

...solche Untersuchungen werden zurzeit aber gerade durchgeführt...

$\mathrm{RB}$

...zweifellos. Aber über eine Soziologie der Lektüre wird man nicht herausfinden können, was eine Lektüre auf der Ebene des Textes ist. Dazu kommt man, glaube ich, über eine, wenn Sie so wollen, erneuerte Linguistik und Psychologie, vielleicht sogar über eine Psychoanalyse, und zwar insofern, als die Psychoanalyse doch gerade dabei ist, sich in diese Richtung zu bewegen. Man könnte auf diese Weise so etwas wie eine allgemeine Theorie des Lesens formulieren oder eine solche vorschlagen. Das wäre bereits ein enormer Fortschritt, wenn man das so anginge und daraus alle Konsequenzen zöge, und zwar dahingehend, dass es in der Weise, wie es ein Subjekt des Schreibens gibt, das man bislang,Autor' genannt hat, nicht wahr, nun im Grunde auch ein Subjekt im vollen Sinne der Lektüre gibt. Man kann sich vorstellen oder davon ausgehen, dass es verschiedene Charaktere oder Züge dieses Subjekts der Lektüre gibt. Man sollte sich dieses Lektüresubjekt nicht als eine endliche Person, als eine genau definierte Identität denken, die entsprechend soziologisch, nicht wahr, durch die Altersklasse, den sozio-professionellen Hintergrund etc. fassbar wäre. 
sujet de la lecture, c'est quelqu'un qui parle le texte aussi d'une certaine façon. Si vous voulez, le schéma émetteur-récepteur se trouve partout. Il ne se trouve pas seulement au niveau grossier du texte mais il se trouve dans chaque acte, dans l'acte d'énonciation et dans l'acte de réception, en quelque sorte, du texte. Cela vous paraît peut-être abstrait, et un peu fumeux disons pour parler franchement, mais il faut bien se dire qu'à partir du moment où on a un petit peu ce fil directeur de chercher à préciser ce que peut être le sujet de la lecture et, au fond, à ne pas le dissocier d'une façon analytique brutale du sujet de l'écriture, alors à ce moment-là, muni de cette sorte de fil conducteur, on pourra peu à peu, mais ce sont des travaux d'assez longue haleine tout de même, n'est-ce pas, on pourra chercher, inventorier, répertorier, classer dans différents types de discours ce que j'appellerai 'les signes véritables de l'interlocution'. On peut arriver en réalité assez vite à saisir des traits absolument concrets qui marquent la trace, si vous voulez, du lecteur dans le texte - ce lecteur qui est déjà, au fond, inscrit dans l'écriture au moment où l'auteur', comme on disait autrefois, produit cette écriture. Vous voyez, ce que je veux dire par là c'est que la vieille dichotomie, la vieille opposition entre l'auteur et le lecteur, était marquée autrefois par une distance très grande entre ces deux termes, et au fond les tentatives de rapprochement qu'on faisait entre le lecteur et l'auteur étaient des tentatives assez verbales et assez pieuses, n'est-ce pas, c'est l'interjection de Baudelaire « Hypocrite lecteur,
Vielmehr ist im Grunde das Lektüresubjekt, so wie wir es jetzt denken, selbst Sprache (langage), wenn Sie so wollen - und in diesem Sinne kann man also sagen: Das Lektüresubjekt ist derjenige, der den Text auch auf eine gewisse Weise spricht. Das Schema Sender-Empfänger findet sich, wenn man so will, überall. Es findet sich nicht nur grob auf der Ebene des Textes, sondern in jedem einzelnen Akt, im Akt des Aussagens und, in gewisser Weise, in jenem der Rezeption des Textes. Das kommt Ihnen vielleicht abstrakt vor oder, um es offen zu sagen, ein bisschen nebulös. Aber zugleich muss man sich doch sagen, dass man von dem Moment an, in dem man ein wenig diesen Leitfaden für den Versuch einer genaueren Erklärung des Lektüresubjekts hat - und um dieses im Grunde nicht analytisch brutal vom Schreibsubjekt zu trennen -, dass also von dem Moment an, in dem man mit einem derartigen Leitfaden ausgestattet ist, man nach und nach das aufsuchen, inventarisieren, erfassen und in verschiedene Diskurstypen gruppieren kann, was ich die ,wahren Zeichen der sprachlichen Interaktion (interlocution) nennen möchte. Es handelt sich dabei allerdings um Arbeiten, die einen langen Atem voraussetzen. Tatsächlich wird es einem recht schnell gelingen, jene sehr konkreten Züge zu erfassen, die, wenn Sie so wollen, die Spur des Lesers im Text markieren - jenes Lesers, der im Grunde bereits in dem Moment in die Schrift und das Schreiben (écriture) eingeschrieben ist, in dem der ,Autor, wie man früher sagte, die Schrift produzierte. Sie sehen, was ich damit 
mon frère ! » c'est-à-dire que tout ce qu'on pouvait imaginer de rapprochement entre l'auteur et le lecteur, c'était d'imaginer une sorte de rapport d'identité, de fraternité entre les deux, alors qu'en réalité il me semble qu'avec les développements de la linguistique, de la littérature et de la psychologie conjuguées, on peut arriver à imaginer ce que j'appellerai une linguistique du lecteur, pas seulement une linguistique de l'auteur, mais une véritable linguistique du lecteur.

GC

Mais est-ce que ça ne suppose pas que nos connaissances en linguistique fassent des progrès préalables qui ne sont pas encore atteints? Nous savons bien quand circule un message littéraire, nous savons bien quand il y a littérature en circulation, c'est toujours dans un même sens, mais nous n'apprécions pas encore la quantité ou la qualité de la littérature qui circule, et ça c'est une gêne sérieuse. sagen will, ist, dass die alte Dichotomie, der alte Gegensatz zwischen Autor und Leser früher gekennzeichnet war durch eine sehr große Distanz zwischen diesen beiden Begriffen; und im Grunde sind die bislang unternommenen Versuche einer Annäherung zwischen Leser und Autor ziemlich oberflächliche und ziemlich fromme Versuche geblieben, nicht wahr. Da ist etwa der Ausspruch von Baudelaire: „Scheinheiliger Leser, mein Bruder!“ (Hypocrite lecteur, mon frère!) - das heißt, dass alles, was man sich an Annäherung zwischen dem Autor und dem Leser vorstellen konnte, darin bestand, sich eine Beziehung der Identität, der Brüderlichkeit zwischen den beiden vorzustellen. Doch in Wirklichkeit scheint es mir, dass man mit den Entwicklungen der Linguistik, der Literaturwissenschaft und der Psychologie, so wie sie miteinander verbunden sind, zur Vorstellung einer Linguistik des Lesers, wie ich sie nennen möchte, gelangen kann. Es geht dann also nicht nur um eine Linguistik des Autors, sondern um eine regelrechte Linguistik des Lesers.

GC

Aber setzt dies nicht voraus, dass unsere Kenntnisse in der Linguistik zuvor Fortschritte gemacht haben, die zurzeit noch nicht erreicht sind? Wir wissen ja, wenn eine literarische Botschaft zirkuliert, wir wissen, wenn Literatur zirkuliert, es geht immer in die gleiche Richtung, aber wir ermessen noch nicht die Quantität und die Qualität der in Umlauf befindlichen Literatur, und das löst ein ernsthaftes Unbehagen aus. 
$\mathrm{RB}$

Oui, mais pour arriver à renouveler le problème de l'auteur et du lecteur, il faut un petit peu, comment dirais-je, séloigner, il faut mettre à une certaine distance peut-être provisoirement le problème du message lui-même.

$\mathrm{Au}$ lieu de faire porter l'accent, par exemple, l'accent de l'analyse, de la critique, de la réflexion, sur les énoncés, il faut le faire porter sur l'énonciation, et une énonciation qui ne doit pas être définie comme un acte psychologique extérieur au texte, mais une énonciation qui est présente au fond à chaque moment du texte et qui a d'ailleurs ses signes dans le texte, n'est-ce pas. Il y a des signes d'énonciation dans le texte et c'est ça qu'il faut repérer. Alors pour ça tout de même, si vous voulez, la linguistique proprement dite, c'est-à-dire dans ses aspects assez particuliers de recherche, ce que j'appellerai la linguistique au niveau des monographies, ne nous renseigne pas peut-être encore beaucoup là-dessus, mais disons que les linguistes qui ont une pensée théorique, qui pensent vraiment en grand sur le langage, pour citer les plus grands, enfin, comme Jakobson, comme Chomsky et comme Benveniste, ce sont des hommes qui tout de même posent le problème de l'énonciation, et pas seulement le problème des énoncés, le problème de l'énoncé. C'est à ce niveau qu'il y a alors une sorte d'intercommunication, d'interaction entre des pensées et des recherches assez différentes; il est certain qu'à partir du moment où la linguistique se pose le problème de l'énonciation, eh bien elle rencontre des
$\mathrm{RB}$

Ja, aber um das Problem des Autors und des Lesers neu zu fassen, muss man sich hier ein wenig, wie ich sagen würde, entfernen, man muss das Problem der Botschaft selbst, vielleicht provisorisch, auf eine gewisse Distanz stellen. Anstatt dass man den Akzent - beispielsweise den Akzent der Analyse, der Kritik, der Reflexion - auf das Ausgesagte legt, muss man den Akzent auf das Aussagen (l'énonciation) legen, und zwar auf ein Aussagen, das man nicht wie einen psychologischen Akt definieren darf, der dem Text äußerlich wäre, sondern auf ein Aussagen, das im Grunde in jedem Moment des Textes präsent ist und das übrigens auch entsprechende Zeichen im Text aufweist, nicht wahr. Es gibt im Text Zeichen dieses Aussagens, und diese muss man ausfindig machen. Allerdings informiert uns darüber die Linguistik im engeren Sinne - das heißt jene Linguistik mit ihren ziemlich spezifischen Forschungsinteressen, die ich Linguistik auf der Ebene von Monografien nennen möchte - vielleicht noch nicht genug. Aber sagen wir, dass die Linguisten, die theoretisch denken, die wirklich grundsätzlich über die Sprache nachdenken um nur die größten zu nennen wie Jakobson, Chomsky und Benveniste -, solche Menschen sind, die sich immerhin dem Problem des Aussagens gestellt haben, also nicht nur dem Problem des Ausgesagten, des Aussagegehalts. Auf dieser Ebene also gibt es eine Art gegenseitiger Kommunikation, eine Interaktion zwischen Überlegungen und Forschungen unterschiedlicher Art. Es ist klar, dass 
recherches par exemple comme celles de Lacan en psychanalyse, c'est évident n'est-ce pas, qui a réfléchi sur le sujet de lénonciation précisément, et comme des recherches comme celles de Sollers en littérature, dont les textes littéraires, surtout les derniers, et surtout le dernier roman, qui s'appelle Drame, est véritablement une réflexion en acte sur les pronoms, sur le 'il' et le 'je', c'est-à-dire sur le matériel même de lénonciation, sur les signes de l'énonciation.

GC

Mais alors comment se manifeste l'action du lecteur sur un texte : Est-ce qu'il collabore, est-ce qu'il reçoit, est-ce qu'il prolonge, est-ce qu'il peut aller jusqu'à se désintéresser complètement du message qui lui est transmis pour, à partir de ce message, élaborer autre chose ? Quel est son type d'action?

\section{$\mathrm{RB}$}

Si vous voulez, je ne vais pas répondre tout de suite à cette question. Il y a un territoire qu'il faudrait explorer avant toute chose. Avant d'explorer ce qui vient de l'énonciateur dans le lecteur, je dirais qu'il faudrait explorer ce qui vient pour ainsi dire a priori et avant toute chose du lecteur dans l'énonciateur...

\section{GC}

...ce que l'auteur a transmis est loin de me paraître le plus important, personnellement... sich von dem Moment an, in dem sich in der Linguistik das Problem des Aussagens (der Aussageweise) stellt, sie wohl auf Forschungen wie zum Beispiel jene von Lacan in der Psychoanalyse stoßen wird, der genau über das Problem des Aussagens nachgedacht hat, das ist offensichtlich, nicht wahr, oder auf Forschungen wie jene von Sollers in der Literatur. Dessen literarische Texte, besonders die letzten - und vor allem der letzte Roman, der Drama heißt - sind tatsächlich eine fortlaufende Reflexion über die Pronomen: über das ,Er‘ und das ,Ich', das heißt über das Material des Aussagens selbst, über die Zeichen des Aussagens.

\section{GC}

Wie nun aber manifestiert sich die Handlung des Lesers in oder an einem Text: Kollaboriert er, empfängt er, verlängert er ihn, kann er so weit gehen, dass er sich an der übermittelten Botschaft überhaupt nicht mehr interessiert zeigt, um, von dieser Botschaft ausgehend, etwas Anderes zu erarbeiten? Um was für einen Typ von Handlung handelt es sich dabei?

\section{$\mathrm{RB}$}

Wenn Sie mir gestatten, antworte ich nicht sogleich auf diese Frage. Es gibt ein Gebiet, das man vor allen anderen Gebieten erforschen müsste. Bevor man untersucht, was im Leser von dem kommt, der sich äußert, würde ich sagen, dass man vielmehr untersuchen müsste, was gewissermaßen a priori und vor allem anderen bereits in dem, der sich äußert, vom Leser kommt...

GC

...was der Autor übermittelt hat, ist fern von dem, was mir persönlich das Wichtigste scheint... 
$\mathrm{RB}$

...oui mais ce que l'auteur a transmis conditionne ce que le lecteur lui-même va faire, je ne dis pas 'va recevoir', mais 'va faire', je veux dire par là, si vous voulez la suite de ma réponse va aussi répondre un peu à votre question -, c'est que quand un auteur se met en acte d'énonciation, quand il écrit, eh bien il est déjà et en même temps non seulement le lecteur de ce qu'il écrit, ce qui serait fort banal à dire, mais le lecteur d'une infinité d'écritures qui l'ont précédé et qui l'entourent. Si vous voulez, maintenant - et je souscris à cette sorte de déplacement de vocabulaire - au lieu de parler d'intersubjectivité' comme on le faisait jusqu'à présent - enfin à propos de la littérature et des rapports du texte et de l'auteur - il vaudrait mieux parler d'intertextualité'. Ce sont les textes qui dialoguent entre eux, et la lecture et l'écriture forment une sorte de tissu dialectique continu. Alors si on pense cela de ce que l'on appelait autrefois l'auteur' et si on arrive à le développer, enfin, à le développer dans une théorie assez fine, je pense que le problème du lecteur sera en partie résolu, parce que le lecteur ne fait rien d'autre que d'être au fond le scripteur virtuel de toutes les écritures qui sont dans le texte. Je crois que lorsque l'on pourra définir avec subtilité et en détail la fonction de ce qu'on appelait autrefois l'auteur' comme étant une sorte de rassembleur des écritures qui l'ont précédé et qui l'entourent, la théorie du lecteur sera beaucoup plus facile à établir, parce que le lecteur lui aussi au fond, dans cette perspective-là et tout au moins à un certain niveau, le
$\mathrm{RB}$

...ja, aber was der Autor übermittelt hat, bestimmt das, was der Leser selbst machen wird - ich sage nicht ,empfangen wird', sondern ,machen wird ' -, womit ich, wenn Sie so wollen (doch der Rest meiner Antwort wird auch ein bisschen auf Ihre Frage antworten) sagen will, dass wenn ein Autor sich daranmacht, etwas auszusagen, wenn er schreibt, er nicht nur zur selben Zeit bereits der Leser dessen ist, was er schreibt, was zu sagen hochgradig banal wäre, sondern er ist der Leser einer Unendlichkeit von Schriften, die ihm vorausgegangen sind und die ihn umgeben. Wenn Sie so wollen, wäre es jetzt besser - und ich befürworte diese Art der Verlagerung des Vokabulars - von der ,Intertextualität' zu sprechen, anstatt von der ,Intersubjektivität', wie man es bislang mit Blick auf die Literatur getan hat. Es geht um Texte, die miteinander in einen Dialog treten, wobei Lesen und Schreiben eine Art fortgesetztes dialektisches Gewebe bilden. Wenn man dies nun für den bedenkt, den man früher ,Autor' nannte, und wenn es einem nun gelingt, dies in einer ausreichend klugen Theorie weiterzuentwickeln, dann denke ich, dass das Problem des Lesers zu einem gewissen Teil gelöst sein wird, weil der Leser nichts anderes macht, als im Grunde der virtuelle Schreiber aller Schriften in einem Text zu sein. Ich denke, dass die Theorie des Lesers viel leichter zu etablieren sein wird, wenn man mit Subtilität und im Detail die Funktion dessen, den man früher ,Autor' nannte, so definiert, dass er eine Art Sammler von Schriften ist, die ihm vorausgegangen 
lecteur n'est rien d'autre qu'une sorte de scripteur virtuel de l'écriture ou de toutes les écritures qui sont dans l'écriture qu'il lit et c'est d'ailleurs pour ça je pense qu'on pourrait dès maintenant esquisser une sorte de théorie - je vais employer un mot qui n'est pas bien noté en philosophie, qui est un mot ancien et assez discrédité -, mais j'aurais envie de parler d'une théorie hédoniste de l'écriture, dans la mesure où je crois profondément que l'écriture, la véritable dimension d'intercommunication de l'écriture, puisque nous parlions de communication, c'est au fond une dimension de plaisir, et pour parler encore plus franchement, et nous l'avons déjà fait ici, une dimension érotique. Et on ne pourrait pas comprendre que le plaisir soit dans la lecture si on ne croyait pas au fond que le lecteur est quelqu'un qui fait, lui aussi, le texte.

\section{GC}

Autrement dit, l'intertextualité ne s'arrête pas à l'action des auteurs, elle se prolonge dans l'action du lecteur.

$\mathrm{RB}$

Exactement.

GC

Mais alors, est-ce que le lecteur reflète, ou est-ce qu'il relaie? sind und die ihn umgeben. Denn der Leser ist in dieser Perspektive und zumindest auf einer gewissen Ebene im Grunde ebenfalls nichts anderes als eine Art virtueller Schreiber der Schrift und von allen Schriften, die in jener Schrift sind, die er liest. Und deshalb denke ich übrigens, dass man ab jetzt eine Art Theorie skizzieren könnte, wobei ich Lust hätte, von dieser Theorie als einer - ich werde ein Wort benutzen, das in der Philosophie nicht viel Beachtung gefunden hat, ein altes und ziemlich diskreditiertes Wort - hedonistischen Theorie der Schrift und des Schreibens (théorie hédoniste de l'écriture) zu sprechen, und zwar insofern, als ich zutiefst glaube, dass die Schrift, die wahre Dimension der gegenseitigen schriftlichen Kommunikation (intercommunication) da wir von der Kommunikation sprachen - im Grunde eine Dimension der Lust ist, und um es noch klarer zu sagen, was wir hier ja schon getan haben: eine erotische Dimension. Man würde wohl nicht verstehen, dass das Lesen lustvoll ist, wenn man nicht im Grunde daran glaubt, dass der Leser, auch er, einer ist, der den Text macht.

GC

Anders gesagt, die Intertextualität hört bei der Handlung der Autoren nicht auf, sie verlängert sich in die Handlung der Leser.

$\mathrm{RB}$

Genau.

GC

Aber reflektiert das der Leser - oder löst er den Autor bloß ab? 
$\mathrm{RB}$

Il ne reflète sûrement pas, il relaie dans la mesure où il reprend, indéfiniment en quelque sorte, laction du scripteur, il est simplement disons un scripteur virtuel. Si je pouvais employer l'image je dirais qu'il est quelqu'un dont le scripteur prend la main et la guide pour au fond écrire en même temps que lui.

GC

Mais si je voulais rendre compte par des schémas de cette action, je crois que ce serait fort difficile et que je me trouverais en présence de figures extrêmement complexes, car l'intertextualité au niveau de l'auteur est tout de même différente de l'intertextualité au niveau du lecteur: j'ai affaire à un flux qui part des auteurs et qui va vers les lecteurs, et il y a tout de même un véritable dialogue établi au niveau des auteurs même quand ils ne s'adressent pas les uns aux autres, chacun est présent à tous les autres...

$\mathrm{RB}$

...oui...

GC

... et tous les autres sont présents à cha-

cun. Lorsque je suis au niveau du lecteur, j'aurai plus de peine à dire que chaque lecteur est présent à tous les autres et que tous les autres sont présents à chacun...

$\mathrm{RB}$

....ah oui, d'ailleurs moi ce n'est pas ce que je dis, je dis simplement qu'il y a un dialogue des textes écrits entre eux, et
$\mathrm{RB}$

Er reflektiert es sicherlich nicht, er löst in dem Maße die Handlung des Schreibers $a b$, oder er nimmt sie gewissermaßen unbestimmt wieder auf, wie er schlicht, sagen wir, ein virtueller Schreiber ist. Wenn ich dafür ein Bild finden müsste, würde ich sagen, dass der Leser jemand ist, der vom Schreiber an die Hand genommen und von ihm dazu geführt wird, im Grunde zur selben Zeit zu schreiben wie er.

GC

Wenn ich mir nun aber die Grundmuster dieser Handlung klarmachen möchte, glaube ich, dass dies hochgradig schwierig wäre und dass ich mich extrem komplexen Figuren gegenübersähe, denn die Intertextualität auf der Ebene des Autors ist doch eine andere als die Intertextualität auf der Ebene des Lesers: Ich habe es mit einem Fluss zu tun, der von den Autoren ausgeht und der zu den Lesern hinführt, und doch gibt es einen tatsächlichen Dialog auf der Ebene der Autoren selbst dann, wenn sie sich nicht aneinander richten, jeder ist allen anderen gegenüber präsent...

$\mathrm{RB}$

...ja...

GC

... und alle anderen sind jedem einzel-

nen präsent. Wenn ich auf der Ebene des Lesers bin, hätte ich größere Mühe zu sagen, dass jeder Leser allen anderen präsent sei und dass alle anderen jedem präsent seien...

$\mathrm{RB}$

...ah ja, doch das ist wohl nicht das, was ich gesagt habe, ich sage schlicht, dass es einen Dialog zwischen geschriebenen 
que devant ce dialogue, qui est l'essentiel et le fondement de la littérature, l'auteur et le lecteur en tant que personnes, en tant que personnes psychologiques, ou sociales, ou douées d'une identité, ont en réalité extrêmement peu d'importance, c'est pour ça que je demande, et je ne suis pas le seul, je demande à ce qu'on substitue peu à peu l'idée de texte - ou l'idée à la rigueur de scripteur - à l'idée d'auteur, pour bien indiquer qu'il ne s'agit plus d'une division psychologique entre des êtres fonctionnellement différents, d'une part l'auteur, alors avec toute sa biographie, ses passions, son talent, son génie d'un côté et d'autre part le lecteur avec toute sa passivité ou dans le meilleur des cas son aptitude au dialogue, ces deux figures étant telles qu'on les imaginait jusqu'à présent pour concevoir l'institution littéraire en quelque sorte. Alors je crois que c'est cette sorte de vue qui est en train de changer, de se modifier.
Texten gibt und dass vor diesem Dialog, der das Wesentliche und das Fundament der Literatur ist, der Autor und der Leser als Personen - als psychologisch oder sozial bestimmte Personen oder als solche, die mit einer Identität versehen sind - in Wirklichkeit extrem wenig Bedeutung haben. Deshalb fordere ich, und da bin ich nicht der Einzige, ich fordere, dass man allmählich die Idee des Autors durch die Idee des Textes - oder allenfalls durch die Idee des Schreibers - ersetzt, um deutlich zu machen, dass es nicht mehr um eine psychologische Unterscheidung zwischen funktional unterschiedlichen Wesen geht: auf der einen Seite der Autor mit seiner ganzen Biografie, seinen Leidenschaften, seinem Talent, seinem Genie - und auf der anderen Seite der Leser mit seiner ganzen Passivität oder im besten Fall mit seiner Eignung zum Dialog. So stellte man sich die beiden Figuren bis zum heutigen Tag vor, um gewissermaßen die Institution der Literatur zu begreifen. Ich glaube nun, dass diese Sicht der Dinge gerade dabei ist, sich zu ändern, sich zu wandeln.

Transkription, Übersetzung und Vorbemerkung von Agathe Mareuge und Sandro Zanetti 